\title{
Magnetic Anisotropy Energy Distribution and Magnetization of CoPt Nanoparticles Encaged in Protein Shell
}

\author{
T. H. Lee ${ }^{1}$, B. J. Suh ${ }^{2}$, and Z. H. Jang ${ }^{1 *}$ \\ ${ }^{1}$ Department of Physics, Kookmin University, Seoul 02707, Korea \\ ${ }^{2}$ Department of Physics, The Catholic University of Korea, Bucheon 14662, Korea
}

(Received 8 December 2016, Received in final form 1 January 2017, Accepted 7 January 2017)

\begin{abstract}
Magnetic properties of CoPt nanoparticles (average size $=\mathbf{2 . 1} \mathbf{n m}$ ) encapsulated in synthesized protein shell have been investigated with SQUID (Superconducting Quantum Interference Device) magnetometer and analyzed by the recently developed non-equilibrium magnetization calculation by our group [T. H. Lee et al., Phys. Rev. B 90, 184411 (2014)]. Field dependence of magnetization measured at 2 K was successfully analyzed with modified Langevin function. In addition, small hysteresis loops having the coercive field of 890 Oe were observed at $2 \mathrm{~K}$. Temperature dependence of magnetization has been measured with zero field cooled (ZFC) and field cooled (FC) protocol with slightly modified sequence in accordance with non-equilibrium magnetization calculation. The analysis on the $M$ vs. $T$ data revealed that the anisotropy energy barrier distribution is found to be very different from the log-normal distribution found in a size distribution. Zero temperature coercive field and Bloch coefficient have also been extracted from the analysis and the validity of those values is checked.
\end{abstract}

Keywords : magnetic nanoparticle, CoPt, non-equilibrium magnetization calculation

\section{Introduction}

It is needless to emphasize the importance of the research on the magnetic nanoparticle systems. There are growing interests on the research and, in many cases, the interests are based on the potential applications in the fields, e.g. magnetic data storage [1,2], magnetic resonance imaging (MRI) contrast agent [3], hyperthermal heating [4, 5]. etc.

But those research and applications have the difficulty due to the distribution of the physical/chemical properties in nanoparticle systems. The distribution is inevitable since the crystalline morphology variations and the size variations among the particles cannot be avoided. Thus it is important to characterize given nanoparticle specimen with the distribution of the physical/chemical properties in mind for the proper application of the nanoparticle system.

Among those magnetic metal and metal alloy nanoparticles, CoPt nanoparticle is known to have large perpendicular magnetic anisotropy and high chemical

CThe Korean Magnetics Society. All rights reserved.

*Corresponding author: Tel: +82-2-910-4768

Fax: +82-2-910-4728, e-mail: zeehoonj@kookmin.ac.kr stability [6]. For the reason, there has been quite an amount of research reports published [7-9]. Among them, the research on the magnetic storage application is most appealing topic for physics and engineering community. For the application, most important physical parameters are magnetic moment and magnetic anisotropy energy barrier. Thus rigorous and in depth characterization of those properties in a magnetic nanoparticle is very important.

In this paper, we report the characterization results of the magnetic properties of $\mathrm{CoPt}$ nanoparticle system. Among the results, magnetic anisotropy energy barrier distribution extraction from the numerical analysis of the temperature dependence of magnetization with special magnetization measurement sequence is discussed.

\section{Sample}

CoPt nanoparticles encaged in hollow protein shell of PepA, a dodecameric bacterial aminopeptidase from Streptococus pneumoniae, was synthesized. Biochemically prepared protein shell has inner diameter of about $6 \mathrm{~nm}$ and external diameter of about $12 \mathrm{~nm}$ [10]. Artificial biomineralization process was performed on the hollow 
protein shell to form CoPt nanoparticle in the internal empty space [6]. Thus formed protein shell - CoPt nanoparticle specimen was analyzed with TEM (Transmission Electron Microscopy) and the TEM image of the nanoparticle specimen revealed that the size of the particle is $2.1 \pm 0.3 \mathrm{~nm}[6]$. Detailed sample synthesis procedure and preliminary characterization results have been described elsewhere $[6,10]$.

\section{Experiment}

The magnetization measurements of the protein shell CoPt nanoparticle specimen were carried out with SQUID (Superconducting Quantum Interference Device) magnetometer (MPMS XL-7, Quantum Design). Temperature dependent magnetization is measured from $2 \mathrm{~K}$ to $300 \mathrm{~K}$ at the external field of 50 Oe. Zero-Field-Cool (ZFC) protocol and Field-Cool (FC) protocol have been modified to meet the requirement of analysis procedure and utilized in data taking. The modification of the measurement sequence is based on the non-equilibrium magnetization calculation model developed previously [11]. The modification is done in the way that waiting time (time needed for the temperature stabilization at each measured temperature) in the measurement sequence is minimized and, unlike the standard sequence, timing information (time stamps of each operation events of SQUID, for example, start of temperature rise, start of measurement, etc.) in the measurement is also recorded.

Field dependent magnetization was measured as functions of field from 0 to $70000 \mathrm{Oe}$ at $2 \mathrm{~K}$. The hysteresis of the magnetization also has been measured at $2 \mathrm{~K}$ by varying the external field in the range from -70000 Oe to 70000 Oe.

\section{Results and Discussion}

As shown in Fig. 1, field dependent magnetization measured at $2 \mathrm{~K}$ does not saturate even at 70000 Oe but shows linearly increasing paramagnetic behavior. Unlike the behavior of simple paramagnets, the field dependence of the magnetization cannot be fitted with simple Langevin function because of the non-saturating behavior. The behavior is quite often observed in magnetic nanoparticle systems and the magnetization curves are sometimes analyzed with modified Langevin function with additional linear susceptibility term [12].

We also have fitted the field dependent magnetization data with the modified Langevin function.

$$
M(H, T)=M_{0}(T)\left[\operatorname{coth}\left(\frac{\mu_{e f f} H}{k_{B} T}\right)-\left(\frac{k_{B} T}{\mu_{e f f} H}\right)\right]+\chi H
$$

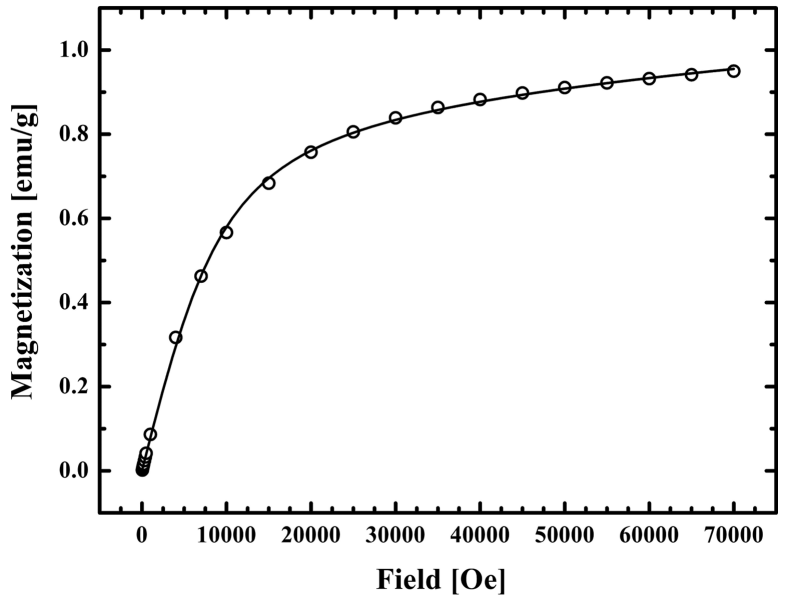

Fig. 1. Field dependence of magnetization measured at $2 \mathrm{~K}$. The solid line is fitting results with modified Langevin function including linear susceptibility term.

From the fitting results, we found that the effective moment is $7.59 \mu_{B}$ and the linear susceptibility, $\chi$, is 1.33 $\times 10^{-6}(\mathrm{emu} /(\mathrm{g} \mathrm{Oe}))$. It is very hard to assign any physical meaning to the values. There have been many attempts to find any physical meaning from the results but the attempts were not so fruitful especially when the system investigated has distributions of physical properties [12].

One direct interpretation of the non-saturating behavior is the existence of extra paramagnetic component other than the main Langevin component and the extra paramagnetic component can be due to impurity or due to the particles with very small anisotropy barrier/blocking temperature of which the behavior is very similar to that of simple paramagnetic particles. Both scenarios can be extended to the idea of the distribution of anisotropy barrier and/or magnetic moment in the magnetic nanoparticle systems.

Previously, we have reported that the temperature dependent magnetization data measured with appropriate measurement procedure can be analyzed with non-equilibrium magnetization calculation to reveal the anisotropy barrier distribution in the ensemble of magnetic nanoparticle system and the validity of the analysis has been confirmed with accurate reproduction of Field-Cooled (FC) magnetization data [11]. Similar analysis has been performed on the CoPt nanoparticle specimen to investigate the anisotropy barrier distribution.

Figure 2 shows Field-Cooled (FC) and Zero-Field-Cooled (ZFC) temperature dependent magnetization measured at the external field of $50 \mathrm{Oe}$. In the figure, we find that the bifurcation temperature, $T_{\text {bifur }}$, the temperature where $\mathrm{FC}$ curve and ZFC curve bifurcate, is different from $T_{\max }$, the temperature at the maximum of the ZFC curve. It is the 


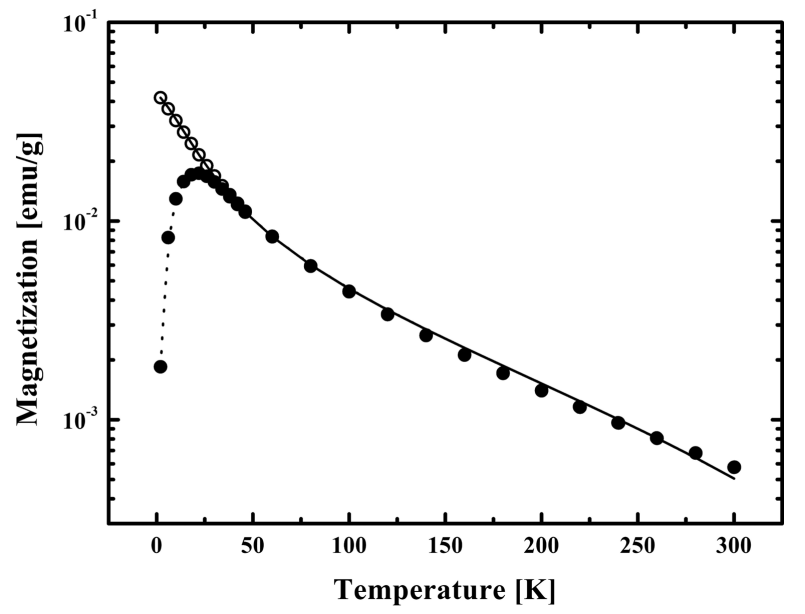

Fig. 2. Temperature dependent magnetization measured at 50 Oe. Open circles represent FC magnetization data and filled circles represent ZFC magnetization data points. Solid line is the best reproduction of FC data with the numerical calculation and dotted line is the numerical calculation results of ZFC data obtained with the same parameter values $\left(H_{C 0}\right.$ and $B)$.

signature of the anisotropy barrier distribution in the sample. If there is not a distribution, both $T_{b i f u r}$ and $T_{\max }$ should coincide.

In the following, we describe brief summary of the back bone of the non-equilibrium magnetization calculation scheme that we have used to analyze the temperature dependent magnetization data. A more detailed description of the scheme is given elsewhere [11].

As in the previous report, we assumed three premises for non-equilibrium magnetization calculation analysis of the experimental data [11]: 1) The particles with the same chemical composition can be categorized uniquely in terms of the zero-field zero-temperature (ZFZT) anisotropy barrier $\left(E_{B 0}^{0}\right)$ : Anisotropy barrier is usually temperature and field dependent. Thus it is better to define an invariant quantity to categorize particles with the definition. 2) Zero field anisotropy energy barrier and magnetic moment of a particle are proportional to each other: It is usually known that magnetic moment and zero field anisotropy barrier of superparamagnetic particles are proportional to each other [13]. 3) The particles are non-interacting: Because of the thick protein shell which covers nanoparticle core, the interaction among the core is very weak. Thus, practically, we can treat nanoparticles to be noninteracting.

One direct and simple logical extension of above premises is the expression for the time dependent magnetization of an ensemble of magnetic nanoparticles at the external magnetic field $H$ and at the temperature $T(M(t$,
$T, H)$ ). The expression is given in terms of the magnetization of single particle with ZFZT anisotropy barrier $\left(E_{B 0}^{0}\right), m\left(E_{B 0}^{0} ; T, H\right)$, and the distribution function of ZFZT anisotropy barrier, $f\left(E_{B 0}^{0}\right)$.

$$
M(t, T, H)=\int_{0}^{\infty} m\left(E_{B 0}^{0} ; t, T, H\right) f\left(E_{B 0}^{0}\right) d E_{B 0}^{0}
$$

Note that the single particle magnetization $m\left(E_{B 0}^{0} ; t, T\right.$, $H$ ) is not an equilibrium quantity rather non-equilibrium quantity. It varies under the influence of external magnetic field, temperature and previous thermodynamic/magnetic history of the ensemble. Time evolution of the single particle magnetization can be described with Bloch equation,

$$
\frac{d m\left(E_{B 0}^{0} ; t, T, H\right)}{d t}=\frac{m_{e q}\left(E_{B 0}^{0} ; T, H\right)-m\left(E_{B 0}^{0} ; t, T, H\right)}{\tau\left(E_{B 0}^{0} ; T, H\right)}
$$

where $\tau\left(E_{B 0}^{0} ; T, H\right)$ is the characteristic relaxation time constant at $T$ and $H$ and it can be calculated with Arrhenius law. We used the Langevin function as a model of the single particle magnetization with temperature dependent magnetic moment of single particle, $\mu(T)$.

$$
m_{e q}\left(E_{B 0}^{0} ; T, H\right)=\mu(T)\left\{\operatorname{coth}\left[\frac{\mu(T) H}{k_{B} T}\right]-\left[\frac{k_{B} T}{\mu(T) H}\right]\right\}
$$

where the temperature dependent single particle magnetic moment is expressed as $\mu(T)=\left(E_{B 0}^{0} / H_{C 0}\right)\left(1-B T^{3 / 2}\right)$. In the expression, $H_{C 0}$ is zero temperature coercive field and $B$ is the Bloch coefficient.

With the non-equilibrium magnetization calculation scheme including the piecewise linear approximation of distribution function of ZFZT anisotropy barrier, we could calculate the ZFC magnetization and the FC magnetization numerically [11] The calculation has been done iteratively to achieve best results which match the experimental data by varying the two free parameters in the scheme, zero temperature coercive field $\left(H_{C 0}\right)$ and Bloch coefficient $(B)$. As final results of the analysis, we could obtain the values of $H_{C O}, B$ and the distribution function of ZFZT anisotropy barrier in piecewise approximated form. The validity of the final results is confirmed by comparing the FC and the ZFC experiment data with numerical calculation results. The other two constants in the calculation are fixed; characteristic time of moment flipping, $\tau_{0}=1 \times$ $10^{-11} \mathrm{~s}$, and characteristic measurement time, $\tau_{M}=10 \mathrm{~s}$.

Before discussing the analysis results, it should be noted that the measurement sequences that we used in the $\mathrm{ZFC}$ and the $\mathrm{FC}$ measurements are different from the usual $\mathrm{ZFC}$ and $\mathrm{FC}$ measurement sequence. In the non- 
equilibrium magnetization calculation, we assumed that temperature is swept continuously without any halt at each measurement data points. In the usual measurement sequence, there are temperature stabilization steps at each measured data points thus there are constant temperature periods in the measurements. We have modified the standard sequence in the way that the constant temperature period is minimized to almost zero.

In Fig. 2, the best results deduced from non-equilibrium calculation are shown as a solid line (FC) and a dotted line (ZFC). The percentage error is very small below 50 $\mathrm{K}$ range and the error gets a little bit bigger as the temperature is increased. We attribute the increase of the error in the high temperature range to comparably large relative experimental error in low magnetization region. But, as is evident in the graph, the overall agreement between the experiment data and the numerical calculation results is excellent.

We have utilized the Langevin function as a model for a single particle equilibrium magnetization. The Langevin function, classical model for paramagnetism, may not be a good model for the magnetism of a single superparamagnetic particle because the effect of anisotropy barrier is predominant at low field and low temperature regime. Even though the expectation, non-equilibrium magnetization calculation revealed almost perfect reproduction of the experiment data in all the temperature range.

The ZFZT anisotropy barrier distribution extracted from the non-equilibrium magnetization calculation and corresponding magnetization measurements is shown in Fig. 3. Most importantly, deduced ZFZT anisotropy barrier distribution is of exponential type which is very different

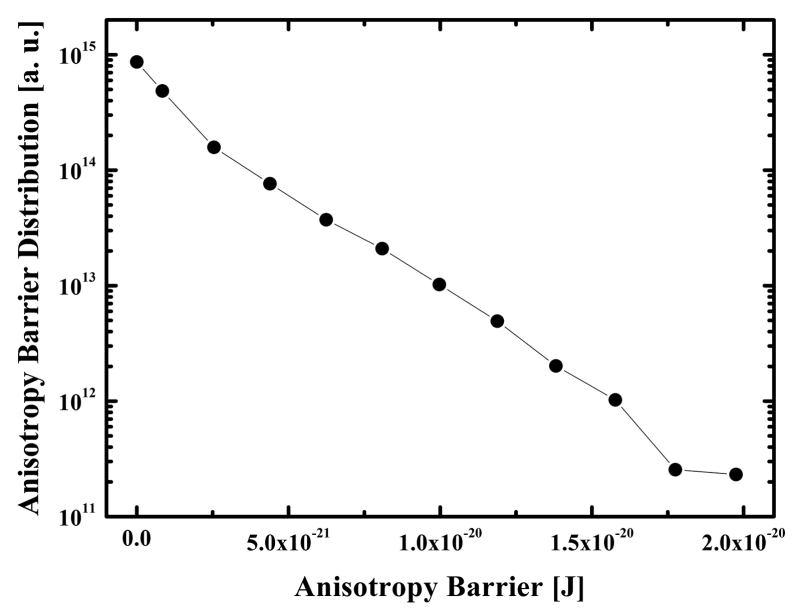

Fig. 3. Extracted ZFZT anisotropy barrier distribution. Overall shape of the distribution is of exponential type which is different from common form of the nanoparticle size distribution, log-normal function. from commonly observed size distribution function in nanoparticles, log-normal function. It is widely accepted that the anisotropy barrier distribution should have of the same shape as the distribution function of the nanoparticle size. The idea is based on the expression, $E_{a}=K V$, that anisotropy barrier $\left(E_{a}\right)$ is proportional to volume of the particle with common anisotropy constant, $K$. But our results show that there is no correlation between the two distributions. One possible explanation of the discrepancy is non-uniform anisotropy constant, $K$. It is highly possible that the crystallinity and morphology in nanoparticles can vary from particle to particle thus non-uniformity in anisotropy constant. But there is not enough information to determine the distribution function of anisotropy constant and/or the correlation between the anisotropy constant and anisotropy barrier.

We also obtained the values of $H_{C 0}$ and $B$ which reproduce best reproduction of the experiment data; $H_{C 0}=$ 956 Oe and $B=9.19 \times 10^{-5} \mathrm{~K}^{-3 / 2}$. Validity of those values has been checked with independent measurement or analysis.

Hysteresis curve is measured at $2 \mathrm{~K}$ and coercive field is found to be 890 Oe. (Fig. 4) Previously, hysteresis was measured at $5 \mathrm{~K}$ and the coercive field was found to be 720 Oe [6]. Considering the observation that coercive field of hysteresis decreases as the temperature is increased [12], it can be noted that the extracted value of zero temperature coercive field $\left(H_{C 0}\right)$ is in harmony with the other coercive field values obtained experimentally.

Above the blocking temperature, ensemble of superparamagnetic particles is in the superparamagnetic state which is similar to usual paramagnetic state but with

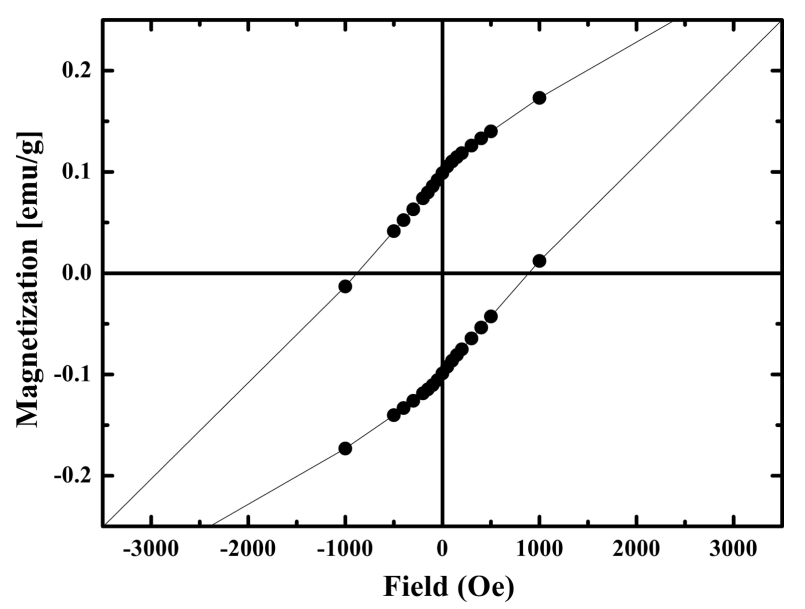

Fig. 4. Hysteresis measured at $2 \mathrm{~K}$. Coercive field of about 890 Oe is observed. Since the hysteresis is so small, only the central section around the origin is shown for coercive field identification. 


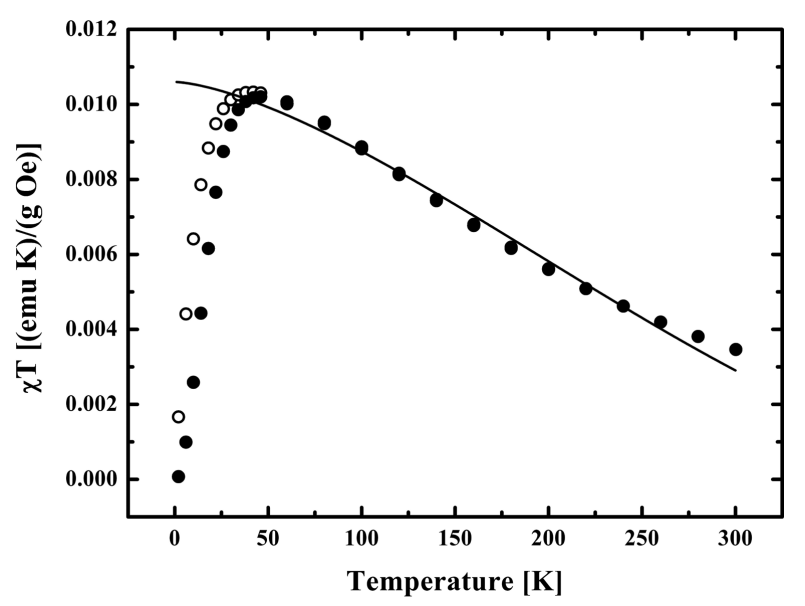

Fig. 5. Temperature dependence of magnetic susceptibility temperature product $(\chi T)$. Solid line is the graph of the Bloch $T^{3 / 2}$ law with Bloch coefficient obtained from the non-equilibrium magnetization calculation (open circle: FC, filled circle: ZFC).

bigger magnetic moments. By using high temperature approximation of Langevin function, magnetic susceptibility - temperature product $(\chi T)$ can be described in terms of magnetic moment of the particles as, $\chi T=\mu^{2}(T)$ / $3 k_{B} \mu_{0} V_{p} \rho \propto \mu^{2}(T)$. Thus we can infer the temperature dependence of the magnetic moment in the $\chi T$ vs. $T$ graph [14]. In ferromagnetic materials, spontaneous magnetization is temperature dependent near Curie temperature and, likewise, magnetic moments of ferromagnetic nanoparticles are also temperature dependent with the same temperature dependence. With Bloch's law, one can write the expression for the temperature dependence as, $\mu(T) \propto\left(1-B T^{3 / 2}\right)$, where $B$ is Bloch coefficient. By using the value of the Bloch coefficient deduced from the non-equilibrium magnetization calculation analysis, we could reproduce temperature dependent magnetic moment and it is compared with experimentally obtained temperature dependence ( $\chi T$ vs. $T$ ) as shown in Fig. 5. Quite satisfactory agreement is observed implying that the value of the Bloch coefficient deduced is in agreement with experiments.

\section{Summary}

We have measured magnetization of CoPt nanoparticles encaged in protein shell at various fields and temperatures. Field dependent magnetization measured at $2 \mathrm{~K}$ could be fitted with modified Langevin function similar to usual magnetization of magnetic nanoparticles. Also small hysteresis was observed at $2 \mathrm{~K}$ and coercive field was found to be 890 Oe which is consistent with previous report and experiment data. Temperature dependence of magnetization showed typical $\mathrm{ZFC}$ and $\mathrm{FC}$ bifurcation and the maximum of ZFC curve is observed at lower temperature than the bifurcation temperature implying the distribution in anisotropy barrier. Measured ZFC magnetization was analyzed with non-equilibrium magnetization calculation yielding the distribution of anisotropy barrier and the values of Bloch coefficient and zero temperature coercive field. By performing iterative calculations, we could reproduce FC data very accurately. Also, validity of the values of Bloch coefficient and zero temperature coercive field deduced is checked against the other experiment data. Finally, extracted distribution of anisotropy barrier distribution is of exponential type which is completely different from the size distribution implying that there is not strong correlation between the size of a CoPt nanoparticle and the anisotropy energy barrier of the particle.

\section{Acknowledgement}

CoPt encapsulated by PepA has been provided by Professor Kyeong Kyu Kim at Sungkyunkwan University $[6,10]$. This research was supported by Basic Science Research Program through the National Research Foundation of Korea (NRF) funded by the Ministry of Education (NRF-2015R1D1A1A01058895).

\section{References}

[1] B. D. Terris and T. Thomson, J. Phys. D: Appl. Phys. 38, R199 (2005).

[2] B. Warne, O. I. Kasyutich, E. L. Mayes, J. A. L. Wiggins, and K. K. W. Wong, IEEE Trans. Magn. 36, 3009 (2000).

[3] H. B. Na, I. C. Song, and T. Hyeon, Adv. Mater. 21, 2133 (2009).

[4] C. D. Kaddi, J. H. Phan, and M. D. Wang, Nanomedicine 8, 1323 (2013).

[5] S. M. Morgan, H. Sohn, and R. H. Victora, J. Appl. Phys. 109, 07B305-1 (2011).

[6] Boi Hoa San, Sanghyun Lee, Sang Hyun Moh, Je-Geun Park, Jung Hee Lee, Hye-Yeon Hwangb, and Kyeong Kyu Kim, J. Mater. Chem. B 1, 1453 (2013).

[7] X. T. Meng, H. C. Seton, L. T. Lu, I. A. Prior, N. T. K. Thanh, and B. Song, Nanoscale 3, 977 (2011).

[8] M. Mandal, B. Das, and K. Mandal, J. Colloid Interface Sci. 335, 40 (2009).

[9] T. Ould-Ely, C. Pan, C. Amiens, B. Chaudret, F. Dassenoy, P. Lecante, M. J. Casanove, A. Mosset, M. Respaud, and J. M. Broto, J. Phys. Chem. B 104, 695 (2000).

[10] D. Kim, B. H. San, S. H. Moh, H. Park, D. Y. Kim, S. Lee, and K. K. Kim, Biochem. Biophys. Res. Commun. 391, 431 (2010). 
[11] T. H. Lee, K.-Y. Choi, G.-H. Kim, B. J. Suh, and Z. H. Jang, Phys. Rev. B 90, 184411 (2014).

[12] Salah A. Makhlouf, F. T. Parker, and A. E. Berkowitz, Phys. Rev. B 55, 14717 (1997).

[13] N. J. O. Silva, V. S. Amaral, L. D. Carlos, B. Rodríguez-
González, L. M. Liz-Marzán, T. S. Berquó, S. K. Banerjee, V. de Zea Bermudez, A. Millán, and F. Palacio, Phys. Rev. B 77, 134426 (2008).

[14] F. Wiekhorst, E. Shevchenko, H. Weller, and J. Kötzler, Phys. Rev. B 67, 224416 (2003). 